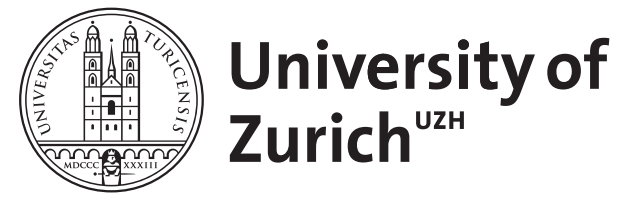

\title{
Analog electronic cochlea with mammalian hearing characterics
}

\author{
Martignoli, S ; van der Vyver, J-J ; Kern, A ; Uwate, Y ; Stoop, R
}

DOI: https://doi.org/10.1063/1.2768204

Posted at the Zurich Open Repository and Archive, University of Zurich ZORA URL: https://doi.org/10.5167/uzh-93203

Journal Article

Published Version

Originally published at:

Martignoli, S; van der Vyver, J-J; Kern, A; Uwate, Y; Stoop, R (2007). Analog electronic cochlea with mammalian hearing characterics. Applied Physics Letters, 91:064108.

DOI: https://doi.org/10.1063/1.2768204 


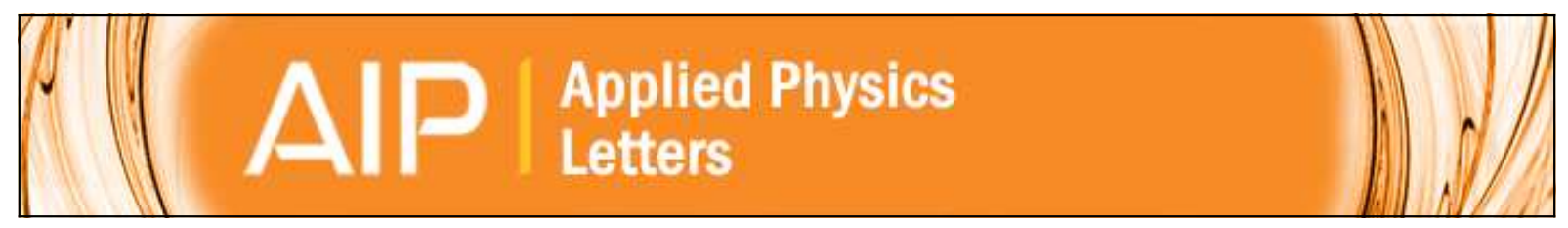

\section{Analog electronic cochlea with mammalian hearing characteristics}

S. Martignoli, J.-J. van der Vyver, A. Kern, Y. Uwate, and R. Stoop

Citation: Applied Physics Letters 91, 064108 (2007); doi: 10.1063/1.2768204

View online: http://dx.doi.org/10.1063/1.2768204

View Table of Contents: http://scitation.aip.org/content/aip/journal/apl/91/6?ver=pdfcov

Published by the AIP Publishing

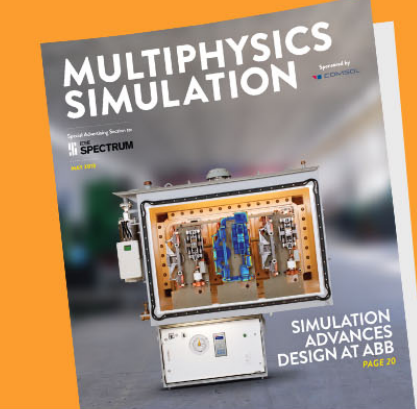

\section{FREE Multiphysics Simulation} e-Magazine

DOWNLOAD TODAY 〉 


\title{
Analog electronic cochlea with mammalian hearing characteristics
}

\author{
S. Martignoli, J.-J. van der Vyver, A. Kern, Y. Uwate, and R. Stoop ${ }^{\text {a) }}$ \\ Institute of Neuroinformatics, University/ETH Zürich, 8057 Zürich, Switzerland
}

(Received 6 June 2007; accepted 12 July 2007; published online 9 August 2007)

\begin{abstract}
Systems close to bifurcations can be used as small-signal amplifiers. Biophysical measurements suggest that the active amplifiers present in the mammalian cochlea are systems close to a Hopf bifurcation. The pure tone and transient signal output of our electronic hearing sensor based on this observation provides output that is fully compatible with the electrophysiological data from the mammalian cochlea. In particular, it reproduces all salient nonlinear effects displayed by the cochlea. (C) 2007 American Institute of Physics. [DOI: 10.1063/1.2768204]
\end{abstract}

The technological implementation of the mammalian hearing sensor, the cochlea, is a long-standing human endeavor, currently of great importance to robotics and medical sciences. In 1863 Helmholtz proposed the existence of a oneto-one correspondence between a position along the cochlea duct and a stimulation frequency to which, at the given position, the response is maximal (the tonotopic principle). Important steps that followed were von Békésy's discovery of traveling waves along the basilar membrane (BM) as the carriers of the auditory information (1928). Gold's conjecture of an active amplification process within the cochlea (1948) was corroborated by the discovery of otoacoustic emissions (1978). Recently, physiological experiments have revealed that the active amplifiers are located in the outer hair cells attached to the BM (Refs. 1 and 2) and that the hearing characteristics vary only moderately among the mammals. It is well established ${ }^{3}$ that systems close to bifurcation instabilities can act as small-signal amplifiers. Detailed physiological measurements ${ }^{4}$ suggest that the instability responsible for active amplification is of Hopf type. ${ }^{5}$ Only this bifurcation correctly captures the basic aspects of mammalian hearing (specific amplification laws with respect to the dynamic range; sharper tuning for lower intensity sounds, the generation of combination tones, two-tone suppression).

In the cochlea, pressure variations generated by incoming sounds are transformed into incompressible and inviscid hydrodynamic waves that, by moving down the cochlea, cause small BM displacements. Using $x$ to denote the distance from the stapes along the unrolled cochlea, the system can be linearly described ${ }^{6}$ by a water-surface wave with fluid depth $h$, density $\rho$, surface mass density $m$, and exponentially decreasing transversal stiffness $E(x)=E_{0} e^{-\alpha x}$. In this description, the wave group velocity $v_{G}$ and the wave number $k$ are related by $v_{G}=\partial \omega / \partial k=[E(x) \rho / 2 \omega][k h+\sinh (k h) \cosh (k h) /$ $\left.(m k \sinh (k h)+\rho \cosh (k h))^{2}\right]$. BM locations $x=x_{c}(\omega)$ respond maximally by (passive) displacements when stimulated at characteristic frequencies $\omega_{c}(x)=\sqrt{E(x) / m}$. This relationship defines the tonotopic map. It can be shown that $k(x, \omega)$ diverges as $\omega$ approaches $\omega_{c}(x)$ and that, as $x$ approaches the point of (passive) resonance $x_{c}(\omega)$ for fixed $\omega$, the traveling wave stalls $\left(v_{G}=0\right)$. Due to dissipative losses, the wave amplitude reaches a maximum at $x<x_{c}(\omega)$. The energy balance equation $\partial e / \partial t+(\partial / \partial x)\left(v_{G} e\right)=0$, for the energy density $e$ together with the ansatz $\partial e / \partial t=-a+d e$, then leads to the co-

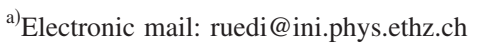

chlea differential equation ${ }^{6}(\mathrm{CDE}) \partial e / \partial x=\left(-1 / v_{G}(x, \omega)\right)$ $\times\left[\left(\partial v_{G}(x, \omega) / \partial x\right)+d(x, \omega)\right] e+\left[a(x, e, \omega) / v_{G}(x, \omega)\right]$, where the local power $a(\cdot)$ supplied by the local active amplification works against the internal viscous losses $d(x, \omega)$ $=4 \nu k(x, \omega)^{2}$, where $\nu$ is the kinematic viscosity. The active amplification results from an array of Hopf-type amplifiers aligned along the BM, each one having its own natural frequency $\omega_{c h}(x)$. Given a forcing frequency $\omega$, the Hopf amplifiers with $\omega_{c h}(x) \approx \omega$ are maximally excited at locations $x_{c h}(\omega)<x_{c}(\omega)$, before viscosity leads to a precipitous decay of the wave amplitude.

For Hopf-type active amplifiers, the active contribution $a$ is derived from an externally forced, $\omega_{c h}$-rescaled Hopf differential equation

$$
\dot{\mathbf{z}}=(\mu+j) \omega_{c h} \mathbf{z}-\omega_{c h}|\mathbf{z}|^{2} \mathbf{z}-\omega_{c h} \mathbf{F}(t), \quad \mathbf{z} \in \mathrm{C},
$$

where $j$ is the imaginary unit. Assuming a 1:1 locking between signal and system, $\mathbf{z}(t)=R e^{j(\omega t+\theta)}$ emerges as the amplified external periodic input $\mathbf{F}=F e^{j \omega t}$, where $\omega_{c h}$ is the natural frequency of the oscillation, and $\mu \in \mathbb{R}$ denotes the Hopf nonlinearity parameter. For $F=0$, Eq. (1) is displays a Hopf bifurcation: For $\mu<0$, the solution $\mathbf{z}(t)=0$ is a stable fixed point, whereas for $\mu>0$, the fixed-point solution becomes unstable and a stable limit cycle of the form $z(t)$ $=\sqrt{\mu} e^{j \omega_{c h} t}$ emerges. For time-varying signals $\mathbf{F}(t)$ it is convenient to put a handle on the response latency, by multiplying the damping term $\omega_{c h}|\mathbf{z}|^{2} \mathbf{z}$ by a factor $\gamma>0$. A nonzero forcing $\mathbf{F}$ then yields $\omega_{c h} F e^{-j \theta}=(\mu+j) \omega_{c h} R-\gamma \omega_{c h} R^{3}-j \omega R$. Evaluation of the squared modulus and introducing the variable $\phi=\omega / \omega_{c h}$ results in $F^{2}=\gamma^{2} R^{6}-2 \gamma \mu R^{4}+\left[\mu^{2}+(1\right.$ $\left.-\phi)^{2}\right] R^{2}$. For $\mu=0$ and close to resonance $\left(\omega=\omega_{c h}\right)$, the response $R=F^{1 / 3}$ emerges, which forces the gain $G=R / F$ $=F^{-2 / 3}$ to increase toward infinity as $F$ approaches zero. For $\mu<0$, maintaining $\omega=\omega_{c h}$, we obtain the response $R=$ $-F / \mu$ for weak stimuli $F$. As $F$ increases, the term $R^{6}$ starts to dominate, and the compressive nonlinear regime is entered, where the differential gain of the system $d R / d F$ decreases with increasing stimulus intensity. Away from resonance, the last term dominates, leading, as $R \approx F /|1-\phi|$, to a linear response, irrespective of the stimulation strength. Intuitively, at nonzero stimulation, the Hopf equation [Eq. (1)] can be interpreted as a nonlinear filter with a tunable gain control ("quality factor") $|\mu|$ and an envelope detector $|\mathbf{z}|^{2}$. As the bandwidth $\Gamma \sim|\mu|$ for $F \leqslant F_{C}$ (and $\Gamma \sim \gamma^{1 / 2} F^{2 / 3}$ for $F>F_{C}$ ), small $|\mu|$ values act as high $Q$ factors (sharp resonances). These properties-unshared by other bifur- 


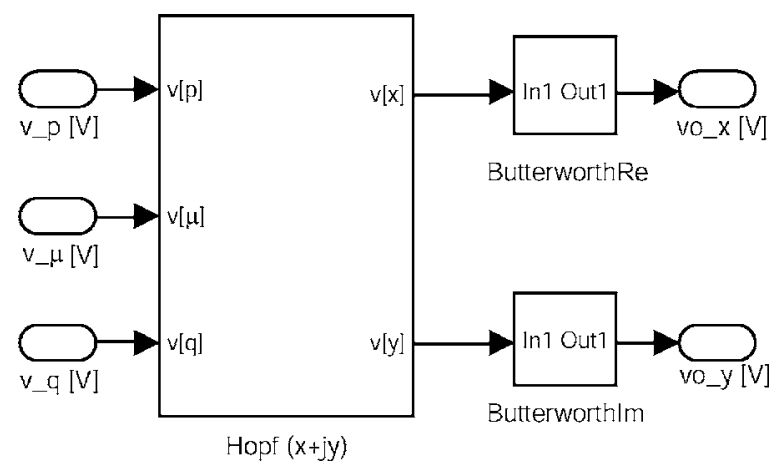

FIG. 1. Section diagram consisting of a Hopf amplifier and two sixth-order Butterworth filters. To form the cochlea, the sections are connected in series. A microphone followed by a Hilbert transform is placed at the beginning of the cascade.

cations ${ }^{7}$ - match the biophysically measured properties of the mammalian cochlea.

For the construction of the hearing sensor, we decomposed the cochlea into $n$ sections of characteristic frequencies $\omega_{s_{i}}, i=1, \ldots, n$, and endowed each one with properties of the passive hydrodynamic behavior and an active Hopf amplifier. The Hopf amplifiers were built in circuitry by using a combination of integrative summers and multipliers, resistors $R$ and a capacitance $C .{ }^{8}$ The responses generated by $\mathrm{CDE}$ (Ref. 6) suggest that the passive part can be modeled as a sixth-order Butterworth circuit. We chose to partition the sixth-order Butterworth filter into three second-order low pass filters. The first filter part has a gain in excess of 1 at the characteristic frequency, leading to an op-amp saturation at large input voltages and small control values. This problem can be compensated for by changing the order of the secondorder circuits. This does not compromise the amplification of small signals, if, following the biological example, the Hopf amplifier preceeds the Butterworth filter. Major challenges were to properly connect the passive/active components toward a section, and the sections toward a cascade representing the entire cochlea. Putting the Hopf amplifier "in front" of the passive unit, and coupling the two units by means of a simplified feedforward coupling, avoids an uncontrollable interaction of the phases of the passive and the active compo- nents, a problem that feedback and open-loop gain amplifications usually struggle with. The corresponding section diagram is shown in Fig. 1.

The cochlea was constructed by serially connecting sections of logarithmically decreasing center frequencies $\omega_{s_{i}}$. In order to build a generic section, it was sufficient to specify the detuning between the passive frequency $\omega_{s_{i}}$ and the Hopf amplifier frequency, $\phi_{s}(i)=\omega_{s_{i}} / \omega_{c h_{i}}<1, i=1, \ldots, n$, where $n$ is the number of sections. This was our first design parameter. The second design parameter was the relationship between the characteristic frequencies of subsequent section frequencies $\Psi(i)=\omega_{s_{i+1}} / \omega_{s_{i}}, i=1, \ldots, n-1$. For the realization of the cochlea as reported below, we chose, for simplicity, the two parameters independent of the section, as $\Psi(i)$ $=\Psi, \phi_{s}(i)=\phi_{s}, \forall i$. For a frequency range to be covered, we use $\Psi$ to determine the number of composing sections, and their characteristic frequencies are evaluated. After choosing a value for the capacitance, the remaining electronic components of the Hopf system are easily obtained, with the electronic gain control $v_{\mu}$ corresponding to the Hopf parameter $\mu$ as the only free parameter, determining both the amplification strength and the tuning width (smaller $|\mu|$ 's lead to larger amplifications combined with sharper tuning widths). Here, we report on an electronic realization based on the design parameters $\phi_{s}=1.05^{-1}$ and $\Psi=0.84$, for which five sections of central frequencies between 1.48 and $2.96 \mathrm{kHz}$ are sufficient to cover one octave in the speech frequency range.

From the constructed electronic device, we measured the amplitude amp $=\left|v_{0}\right|$ generated in response to pure tones of distinct input frequencies and amplitudes. The comparison of the measurements after the first and the second section demonstrates that by passing through the sections, the signal is gradually shaped. After a few (here: five) sections, the response attains its characteristic form, see Fig. 2. The response is taken at two values of the Hopf parameter $\mu$ in order to demonstrate the influence $\mu$ has on the amplification strength and how it may generate a moderate discretization effect, if the amplification in relation to the distance (expressed by $\Psi$ ) between the sections is too large. When moving with the measurement point down the cochlea, a change (a)
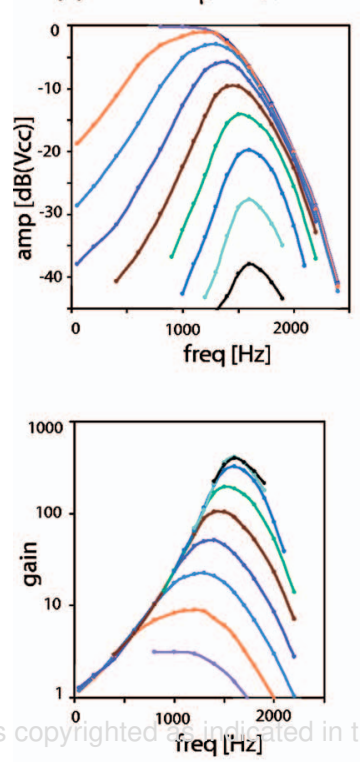

(b)
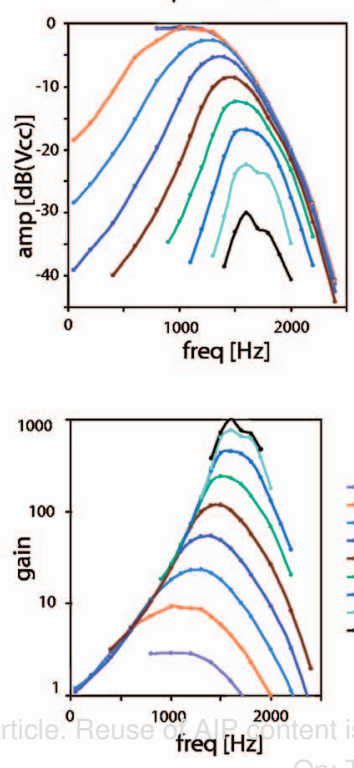

(c)
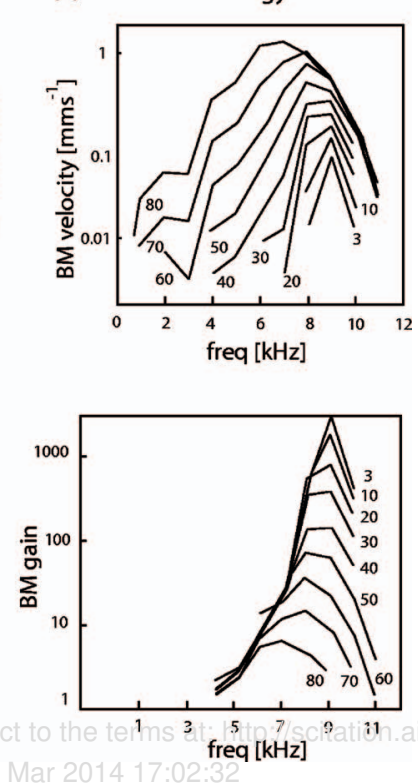

FIG. 2. (Color) (a, b) Steady-state response (upper panels) and gain (lower panels) for Hopf parameters $\mu=-0.1$, -0.05 . Central frequency: $1.48 \mathrm{kHz}$. (c) Physiological measurements (Ref. 9). The device's response is closer to the electrophysiological response than previous modeling results (Refs. 6, 10, and 11). 

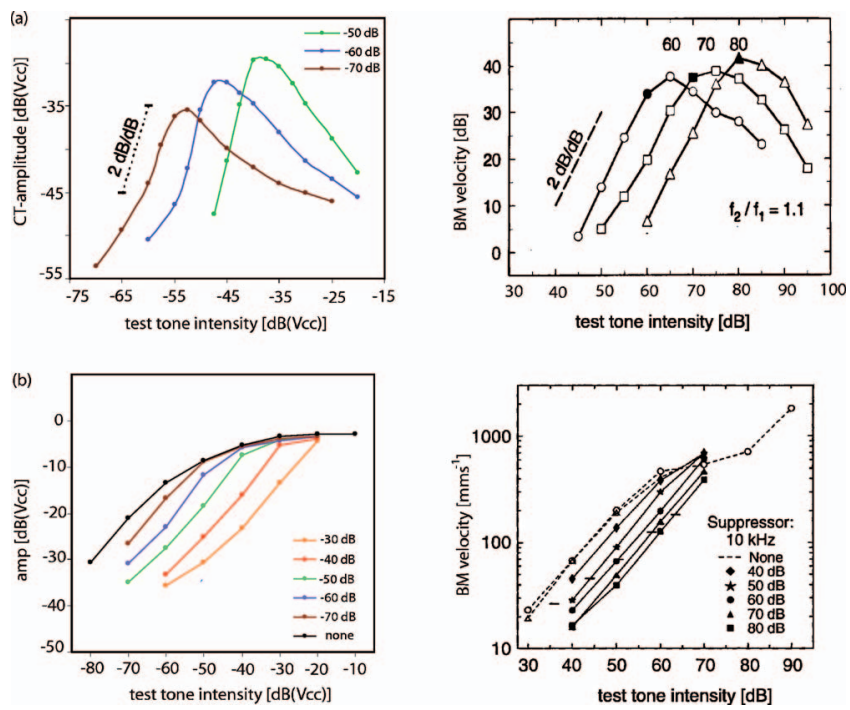

FIG. 3. (Color) Salient nonlinear phenomena of hearing: (a) Combination tone, (b) two-tone suppression. Left: electronic cochlea $(\mu=-0.05$, central frequency of $1.48 \mathrm{kHz}$ ), right: physiological measurements (Refs. 13 and 14). The results also closely match with the theoretical predictions (Ref. 15).

of the response shape is observed that is very close to the one exhibited by the biological example. ${ }^{12}$ The response phases lag increasingly as the frequency of stimulation increases. Two-tone suppression and combination-tone generation, as the salient nonlinear phenomena of mammalian hearing ${ }^{13-15}$ and presumably important for obtaining a "full" sound impression, are reliably reproduced by the device, see Fig. 3. The response to transient, broadband stimulation (Fig. 4, for click stimulations), reveals a close match with the biophysi-

(a)

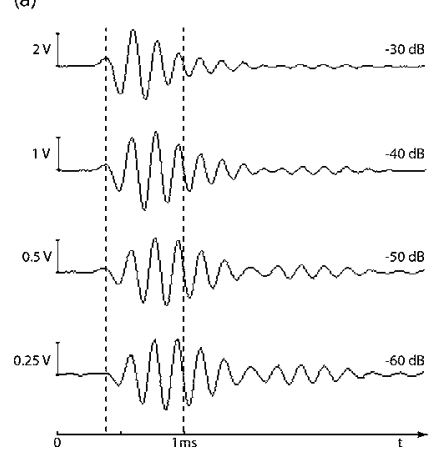

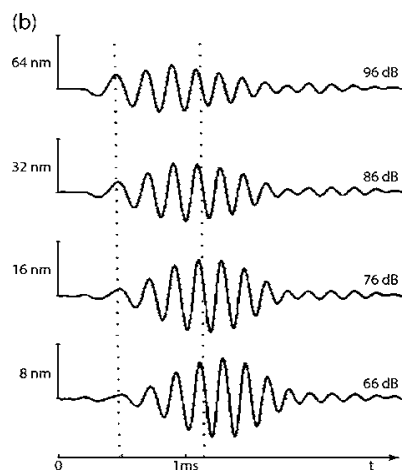

FIG. 4. Response to broadband transient stimulation (clicks): (a) hardware $(\mu=-0.05$, central frequency of $5 \mathrm{kHz}$ ), (b) physiological measurments (chinchilla, $5.5 \mathrm{kHz}$, from Ref. 16). cal measurements. ${ }^{16}$ All basic biophysical experiments with transient signals ${ }^{16}$ are reproduced with great fidelity. For example, the instantaneous frequency response for different stimulus levels reproduces the logarithmic increase towards the steady-state response near the central frequency. The particular section design renders the device noise robust: Despite using standard electronic components, around the central frequency inputs down to $-90 \mathrm{~dB}[\mathrm{Vcc}]$ are detectable. At $\mu \approx-0.1$, the measured dynamic range exceeds $60 \mathrm{~dB}$.

The device thus provides an extremely sensitive and robust hearing sensor, where salient nonlinear signal processing characteristics (compressive nonlinearity, high sensitivity, two-tone suppression, combination-tone generation) are naturally comprised and where steady-state as well as transient signal response characteristics are fully compatible with the electrophysiological measurements (supporting supplementary data available). By following closely the biological example, it is also in the nature of the device that the response could be optimized by a response-dependent value of $\mu$ (increasing $|\mu|$ with signal onset). More importantly, on slower time scales, via the auditory neuronal feedback loop, $\mu$ can be tuned in order to enhance desired and to suppress unwanted signal components.

This work was supported by SNF Grant No. 205321108427. Y.U. acknowledges the support by the Japanese Ministry of Education and the University of Tokushima.

${ }^{1}$ W. E. Brownell, C. R. Bader, D. Bertrand, and Y. de Ribaupierre, Science 227, 194 (1985)

${ }^{2}$ L. Robles and M. A. Ruggero, Physiol. Rev. 81, 1305 (2001).

${ }^{3}$ K. Wiesenfeld and B. McNamara, Phys. Rev. Lett. 55, 13 (1985).

${ }^{4}$ C. D. Geisler, From Sound to Synapse (Oxford University Press, Oxford, UK, 1998), p. 65.

${ }^{5}$ V. M. Eguíluz, M. Ospeck, Y. Choe, A. J. Hudspeth, and M. O. Magnasco, Phys. Rev. Lett. 84, 5232 (2000).

${ }^{6}$ A. Kern and R. Stoop, Phys. Rev. Lett. 91, 128101 (2003).

${ }^{7}$ E. D. Surovyatkina, Y. A. Kravtsov, and J. Kurths, Phys. Rev. E 72, 046125 (2005).

${ }^{8}$ R. J. Smith and R. C. Dorf, Circuits, Devices and Systems (Wiley, New York, 1991).

${ }^{9}$ M. A. Ruggero, Curr. Opin. Neurobiol. 2, 449 (1992).

${ }^{10}$ M. O. Magnasco, Phys. Rev. Lett. 90, 058101 (2003).

${ }^{11}$ T. Duke and F. Jülicher, Phys. Rev. Lett. 90, 158101 (2003).

${ }^{12}$ M. A. Ruggero, S. S. Narayan, A. N. Temchin, and A. Recio, Proc. Natl. Acad. Sci. U.S.A. 97, 11744 (2000).

${ }^{13}$ L. Robles, M. A. Ruggero, and N. C. Rich, J. Neurophysiol. 77, 2385 (1997).

${ }^{14}$ M. A. Ruggero, L. Robles, and N. C. Rich, J. Neurophysiol. 68, 1087 (1992).

${ }^{15}$ R. Stoop and A. Kern, Phys. Rev. Lett. 93, 268103 (2004).

${ }^{16}$ A. Recio and W. S. Rhode, J. Acoust. Soc. Am. 108, 2281 (2000). 